\title{
Influence of leaf shape on the scaling of leaf surface area and length in bamboo plants
}

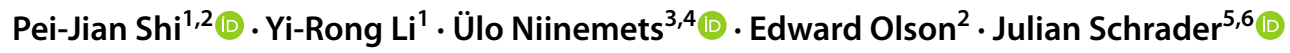

Received: 11 December 2019 / Accepted: 31 October 2020 / Published online: 29 November 2020

(c) The Author(s) 2020, corrected publication 2020

\begin{abstract}
Key message Using more than 10,000 bamboo leaves, we found that the scaling between leaf surface area and length follows a uniform power law relationship mainly relying on the degree of variation in leaf shape (reflected by the ratio of leaf width to length).

Abstract A recent study based on leaf data of different plant taxa showed that the scaling of leaf surface area $(A)$ with linear leaf dimensions was best described by the Montgomery equation (ME) that describes $A$ as the product of leaf length $(L)$ and width $(W)$. Following from ME, a proportional relationship between $A$ and the square of $L$ has been proposed, but the validity of this simplified equation strongly depends on leaf shape (W/L ratio). Here, we show that the simplified equation can be applied to a group of closely related plants sharing a similar $W / L$ ratio with low degree of uncertainty. We measured $A, L$ and $W$ of more than 10,000 leaves from 101 graminoid taxa (subfamily Bambusoideae) having similar elongated leaf shapes. We found that ME applies to the leaves of all bamboo taxa investigated. The power law equation that was used to describe a scaling relationship of $A$ vs. $L$ also predicted leaf area with high accuracy, but the variability measured as the root-mean-square error (RMSE) was greater than that using ME, indicating that leaf width also plays an important role in predicting leaf area. However, the dependence of the prediction accuracy of $A$ on $W$ is intimately associated with the extent of the variation in $W / L$ ratio. There was a strong positive correlation $(r=0.95$ for the 101 bamboo taxa) between RMSE and the coefficient of variation in $W / L$ ratio. Thereby, our results show that leaf area of bamboo plants can be calculated by the product of $L$ and $W$ with a proportionality coefficient ranging from 0.625 to 0.762 , i.e., the leaf area of bamboo leaves approximately equals $70 \%$ of a rectangular area formed by $L$ and $W$. The pooled data of $\ln (A)$ vs. $\ln (W L)$ of 101 bamboo taxa were located on or very close to the regression line without being affected by interspecific differences. However, interspecific differences in $W / L$ ratio largely affected the scaling relationship of $A$ vs. $L$, which led to large deviations of the data of $\ln (A)$ vs. $\ln (L)$ from the regression line. This implies that the square relationship between $A$ and $L$ does not apply to all species even if those are very closely related taxa.
\end{abstract}

Keywords Montgomery equation $\cdot$ Power law $\cdot$ Principle of similitude $\cdot$ Ratio of leaf width to length $\cdot$ Scaling . Bambusoideae

Communicated by H. Roaki Ishii.

Electronic supplementary material The online version of this article (https://doi.org/10.1007/s00468-020-02058-8) contains supplementary material, which is available to authorized users.

Julian Schrader

jschrad@uni-goettingen.de

Extended author information available on the last page of the article

\section{Introduction}

Many leaf measurements and scaling relationships including leaf area, leaf dry mass per unit area, specific leaf area, and leaf thickness are closely associated with the photosynthetic capacity of plants (Milla and Reich 2007; Niinemets et al. 2007; Niklas et al. 2007), thereby being important indices for plant species ecological strategy (Wright et al. 2004). Among the allometric scaling relationships of leaves, the power law relationship between leaf dry mass and area reflects the tradeoff between photosynthetic returns and investments in leaf 
physical supporting structures (Niinemets et al. 2007; Niklas et al. 2007).

According to the principle of similitude proposed by Thompson (1917), the area of an object is generally proportional to the square of its length (i.e., a power law relationship with a scaling exponent of 2). However, several empirical studies have suggested that leaf area and length do not follow a power law relationship with a scaling exponent of 2 , whereas the estimated scaling exponent could be smaller or greater than 2 (Verwijst and Wen 1996). In a study on six plant taxa with different leaf shapes (e.g., ten populations of Parrotia subaequalis (H. T. Chang) R. M. Hao \& H. T. Wei, 5 species of Lauraceae, 2 species of tulip trees (Liriodendron tulipifera $\mathrm{L}$. and Liriodendron chinense (Hemsl.) Sarg.) and their hybrids, 5 species of Oleaceae, 12 species of Bambusoideae, and 12 species of Rosaceae), Shi et al. (2019a) reported that the leaf area was predicted with high accuracy by the product of leaf width and length. Whether the power law relationship with a scaling exponent of 2 between leaf area and length was valid mainly depended on the degree of variation in the ratio of leaf width to length ( $W / L$; Shi et al. 2019a). The correlation coefficient between $A$ and $L$ was at most 0.85 , and the smaller the coefficient of variation in $W / L$ ratio, the stronger was the power law relationship with a scaling exponent of 2 between $A$ and $L$ (Shi et al. 2019a). However, while leaf length is generally defined as the distance from leaf apex to the connection point between blade and leaf petiole, the definition of leaf width is not straightforward for several species with complex leaf shapes like those of tulip trees (Liriodendron spp.). Thus, the variation in the complexity of leaf shapes may have blurred the influence of $W / L$ ratio variation on the scaling relationship between leaf area and length for this dataset.

In the present study, we test whether the influence of the $W / L$ ratio variation in estimating leaf area is driven by differences in leaf shape between species. Therefore, we used $>10,000$ leaves of 101 taxa within Bambusoideae (a subfamily of Poaceae) that share very similar leaf shapes to further examine the influence of leaf-shape variation on the validity of the power law relationship with a scaling exponent of 2 between leaf area and length. In Bambusoideae, the leaf-shape variation is mainly reflected in the $W / L$ ratio (Shi et al. 2015, 2018, 2019b), and accordingly, we expected to obtain more robust scaling relationships among $A$ and $L$, and conclusively test the power law relationship with a scaling exponent of 2 .

\section{Materials and methods}

\section{Data sources}

We measured 101 taxa, cultivars, forms, and varieties of bamboo (in total 80 bamboo species, independent of cultivar, form and variety, from 17 genera of Bambusoideae). Eightynine bamboo taxa were collected from Nanjing City, Jiangsu Province, China; 8 bamboo taxa were collected from Tongzhi County, Zunyi City, Guizhou Province, China; 4 bamboo taxa were collected from Kunming City, Yunan Province, China (detailed collection information can be found in Table 1 in Lin et al. 2020). For each bamboo taxon, we measured 90-110 leaves. Leaf length was defined as the distance from leaf apex to the connection point of leaf blade and petiole, and leaf width was defined as the maximum distance of any two points on the blade edge perpendicular to the straight line connecting leaf apex and the attachment point of the petiole. The planar coordinates of the leaf outline were extracted by a Matlab procedure developed by Shi et al. (2018); leaf length, width, and area were calculated by a script based on the statistical software R (version 3.2.2; $\mathrm{R}$ Core Team 2015). The relevant Matlab procedure and $\mathrm{R}$ script used can be found in the online supplementary materials published in Shi et al. (2018) and Su et al. (2019). All raw leaf data can be found and freely downloaded from Lin et al. (2019; datal file).

\section{Analyses}

We used the Montgomery equation (ME) to describe the proportional relationship between leaf area $(A)$ and the product of leaf length $(L)$ and width $(W)$, which was first proposed by Montgomery (1911) to calculate the leaf area of corn (Zea mays):

$A=k \times L \times W$

where $k$ is a proportionality coefficient (note that we refer to this equation as the Montgomery equation and to $k$ as the Montgomery parameter through the text, also to make our work better comparable to the larger body of literature on this topic (e.g., Shi et al. 2019a, b, Yu et al. 2020)). To normalize the distribution of the response variable, we logtransformed both sides of Eq. (1) as:

$\ln (A)=a+\ln (L W)$

Here, $a$ is a constant to be fitted and $k=\exp (a)$.

To explore the scaling relationship between $A$ and $L$, we used the linearized equation:

$\ln (A)=q+d \times \ln (L)$

where $q$ and $d$ are both fitting constants. We also fitted the relationship between $\ln (A)$ and $\ln (L)$ using the following linearized form:

$\ln (A)=c+2 \times \ln (L)$

where the slope is fixed to 2 according to the principle of similitude. That is, the area of an object is empirically 
proportional to the square of its length (i.e., the principle of similitude proposed by Thompson (1917)), and $c$ is a constant to be fitted. The equations were fitted by minimizing the sum of the squares between the measured and predicted values. To measure the goodness of fit, the root-mean-square error (RMSE) was used. RMSE equals the square root of the mean residual square. We also used the correlation coefficient $(r)$ to measure the strength of a linear relationship between the predicted and measured values.

We first examined whether $A$ is proportional to the product of $L$ and $W$. If this proportional relationship holds true, we then considered whether the extent of the variation in $W / L$ ratio can influence the scaling exponent of $A$ vs. $L$. It is apparent that the larger the variation in $W / L$ ratio, the larger is the degree of the deviation of the scaling exponent of $A$ vs. $L$ from 2 (on the condition that ME well describes the relationship between $A$ and $L W$ ). Thus, we plotted the root-mean-square error in the linear fit (to the data of the logarithm of $A$ vs. that of $L$ with a slope of 2) against the coefficient of variation in $W / L$ ratio to test whether there was a positive correlation between these two measures.

\section{Results and discussion}

We found significant differences in the $W / L$ ratio among the 101 bamboo taxa (Fig. 1a; see data3 file in Lin et al. [2019]). Even within the genus Pleioblastus, there were still two taxa that had narrower leaves than the remaining ten taxa (Fig. 1a). The ME provided valid estimates for each of the 101 taxa, and the correlation coefficients fell into a range of 0.984-1 (Fig. 2; see also Supplementary Fig. S1-S101). The estimated Montgomery parameters for the 101 taxa fell into a narrow range of $0.62-0.78$ (Fig. 2), and at the subfamily level the Montgomery parameter equaled 0.696 with a $95 \%$ confidence interval $(0.695,0.697)$. Whether at the individual taxon (i.e., here referring to species, cultivars, forms, and varieties) or subfamily level, there was a significant and strong proportional relationship between leaf area and the product of leaf length and width. Although there were large differences in $W / L$ ratios among different species, the product of leaf length and width showed a stable proportional relationship with leaf area for the pooled data (Fig. 1a, b). The pooled data of $\ln (A)$ vs. $\ln (L W)$ were located on or very close to the regression line with a slope of 1 , which did not show any evidence for the influences of the differences in the affinities of species on the proportional relationship (Fig. 1b). However, the data of $\ln (A)$ vs. $\ln (L)$ seriously deviated from the regression line with a slope of 1.8 for some species, especially for the genus Pleioblastus, and this estimated slope was significantly different from 2 as suggested by the principle of similitude (Fig. 1c). There was no correlation between the Montgomery parameter and the mean
$W / L$ ratio $(r=-0.13 ; P=0.1942>0.05)$, which showed that the proportional relationship between $A$ and $L W$ was not affected by the $W / L$ ratio. Further, we found a significant scaling relationships between leaf area and length, both at the individual species and subfamily levels (Fig. 1c; Figs. $\mathrm{S} 102-202)$. However, the average \pm SE correlation coefficient $(r)$ of $\ln (A)$ vs. $\ln (L)$ relationships $(0.9556 \pm 0.0234)$ was lower than that of $\ln (A)$ vs. $\ln (L W)(0.9940 \pm 0.0030)$. This indicates that using leaf length alone as a predictor for leaf area could cause a larger error relative to using the product of leaf length and width. There was a very strong correlation between the coefficient of variation and the RMSE value obtained using a power function with a scaling exponent of 2 to fit the leaf area vs. length (Fig. 3, $r=0.949, P<0.01$ ), and only one estimation (in Dendrocalamus sinicus L.C. Chia et J. L. Sun) deviated largely from the regression line. Shi et al. (2019a) obtained an $r$ value of 0.85 for 47 datasets belonging to six families of plants in that study. The results of the present study demonstrated that for similar leaf shapes within the same subfamily, the validity of a power law relationship with a scaling exponent of 2 between leaf area and length is driven by the degree of variation in $W / L$ ratio.

Shi et al. (2015) and Lin et al. (2016) found that the leaf shapes of bamboos could be described by a polar coordinate equation with two parameters, which is a special simplified case of the original Gielis equation (Gielis 2003) that effectively simulates many symmetrical geometric shapes for abiotic and biotic objects. Shi et al. (2018) confirmed that the two variables in that coordinate equation could be expressed by leaf length and width. Thus, the leaf shape differences among different bamboo species could be measured by $W / L$ ratio. The interspecific variation in leaf shape of bamboos can be further expressed by the coefficient of variation of $W / L$ ratio. Although the current study has shown significant variations in $W / L$ ratios among different bamboo taxa, the variations affect the proportional relationship of leaf area vs. the product of leaf length and width only to a minor degree (Fig. 2b). Our data show that the leaf area is approximately equal to $70 \%$ of the area of a rectangle with its length and width being leaf length and width. This conclusion confirms the results obtained for 20 bamboo species in Shi et al. (2019b). However, that study lacked sufficient data to analyze the correlation between the coefficient of variation in $W / L$ ratio and the goodness of fit for a power law relationship with a scaling exponent of 2 from many species of a large family (or subfamily) with similar leaf shapes. Due to the similarity in leaf shapes, bamboo leaves have obtained a fairly high correlation coefficient between RMSE (obtained from fitting the power law function with a scaling exponent of 2 between leaf area and length) and the coefficient of variation in $W / L$ ratio. This study further verified our hypothesis that whether the principle of similitude (i.e., the hypothesis that the area of 


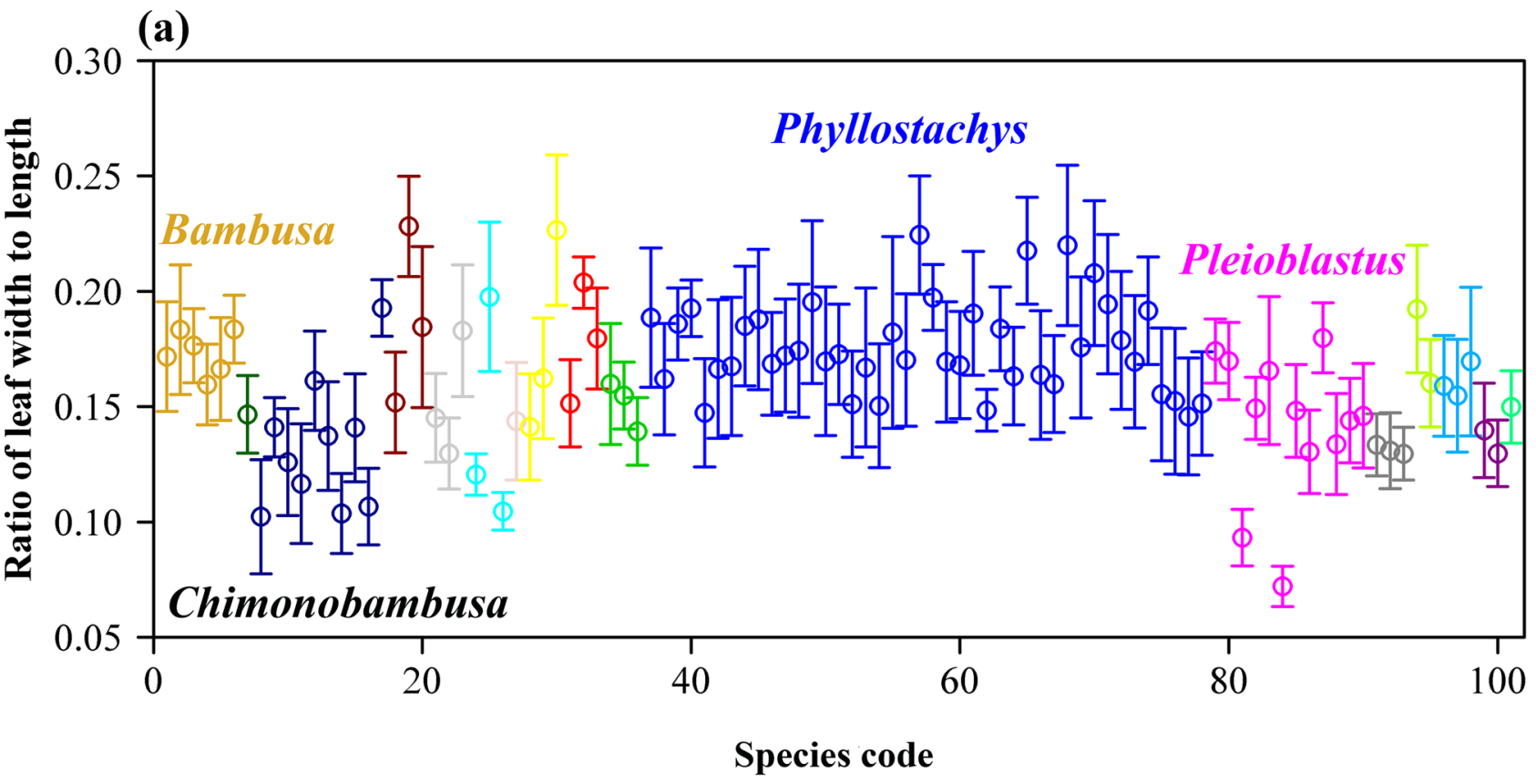

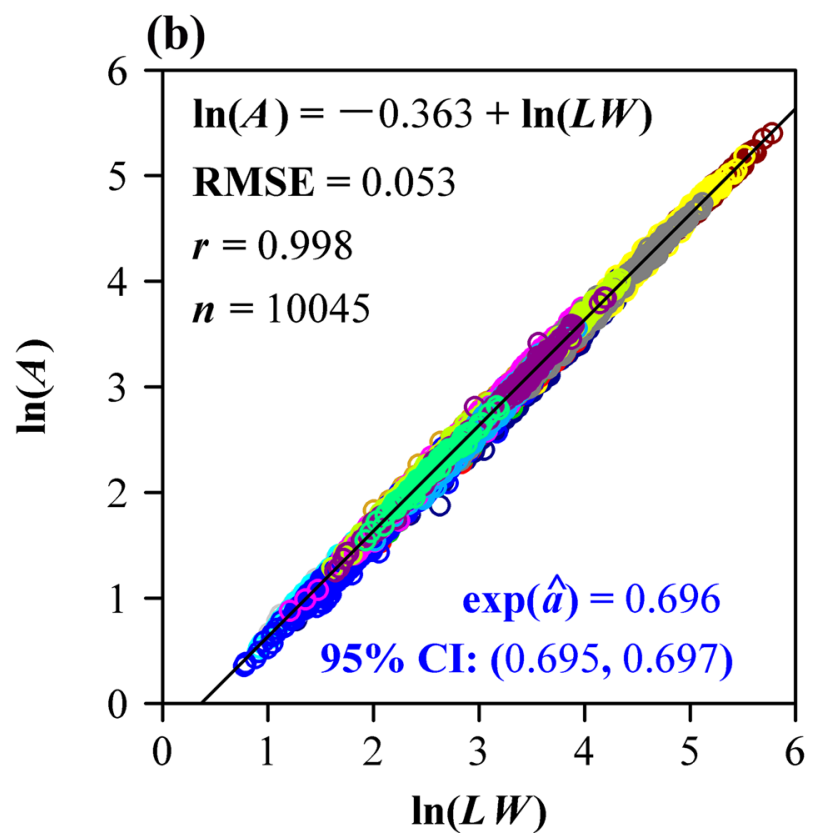

Fig. 1 Ratio of leaf width $(W)$ to length $(L)$ and the scaling relationships between leaf area $(A)$ and combinations of $L$ and $W$. a Ratios of leaf width to length for 101 bamboo taxa. Scientific names of taxon codes are provided in the Supplementary Table S1. Open circles represent the mean $W / L$ ratios; the error bars represent the standard deviations; different colors represent different genera of Bambusoideae. b The proportional relationship between leaf area and the product of

an object is proportional to its length squared) holds true in leaves mainly relies on the degree of variation in $W / L$ ratio based on our results from 101 bamboo taxa, since the Montgomery equation has demonstrated its general applicability in predicting leaf area.

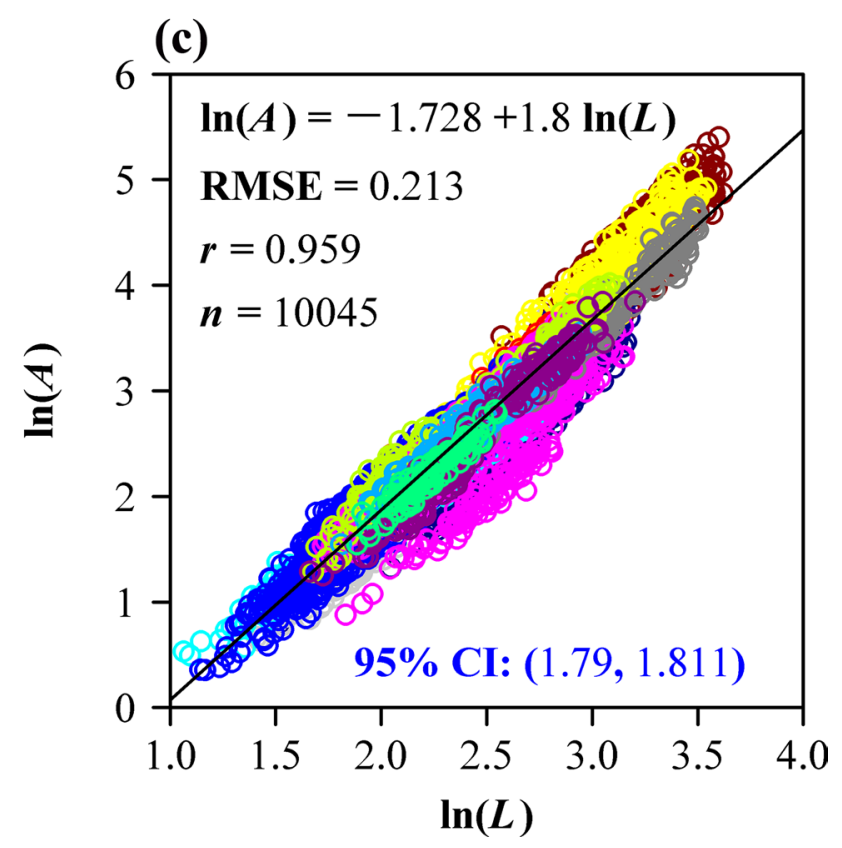

leaf length and width. $\exp (\widehat{a})$ is the estimate of the proportionality coefficient in the Montgomery equation (where the caret on the head of a represents "the estimate of"), and 95\% CI provides 95\% confidence intervals of the proportionality coefficients. $\mathbf{c}$ The scaling relationship between leaf area and length. 95\% CI in this panel represents the $95 \%$ confidence intervals of the slope

Environmental factors, especially temperature, could significantly affect leaf shapes (Peppe et al. 2011). The scaling relationship between the leaf area and length may have been influenced by differences in environmental conditions. For example, D. sinicus (Fig. 3) is an outlier that seriously 


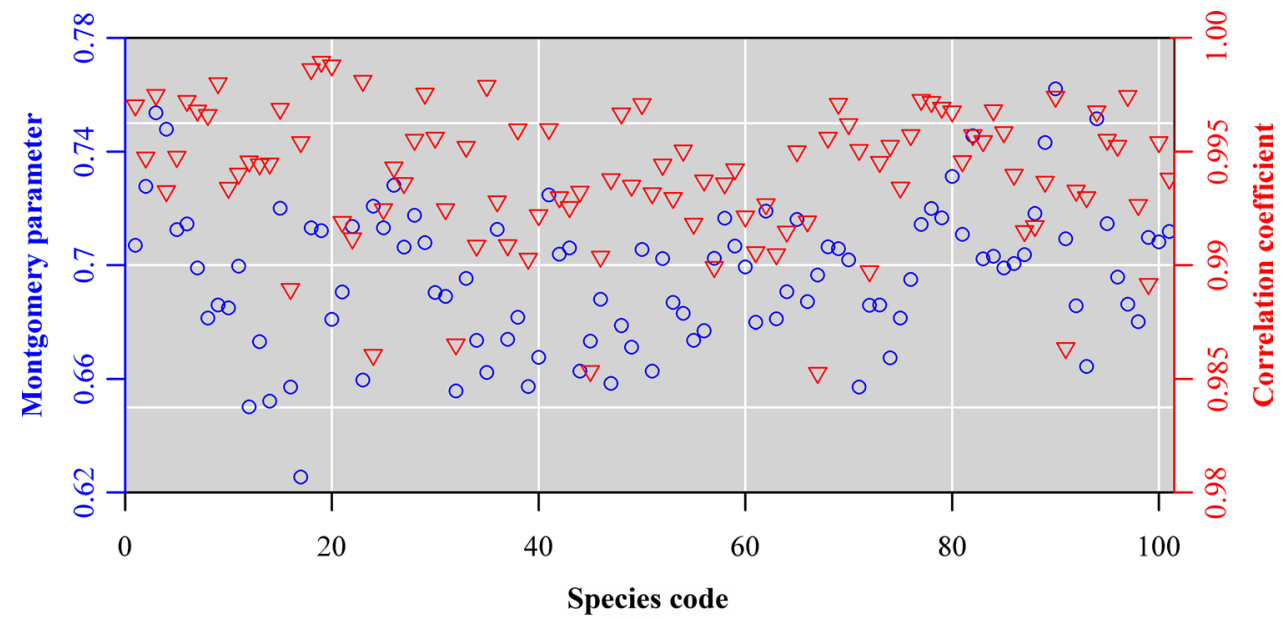

Fig. 2 Estimated Montgomery parameters (i.e., the proportionality coefficient in the Montgomery equation) and the corresponding correlation coefficients of the linear equations for 101 bamboo taxa. Each small open circle represents the estimated Montgomery parameter of a bamboo taxon, and each open inversed triangle represents the correlation coefficient between leaf area and the product of leaf length and width for the bamboo taxon. The order of dataset code on the $x$-axis is the same as that in Table 1 published in Lin et al. (2020)

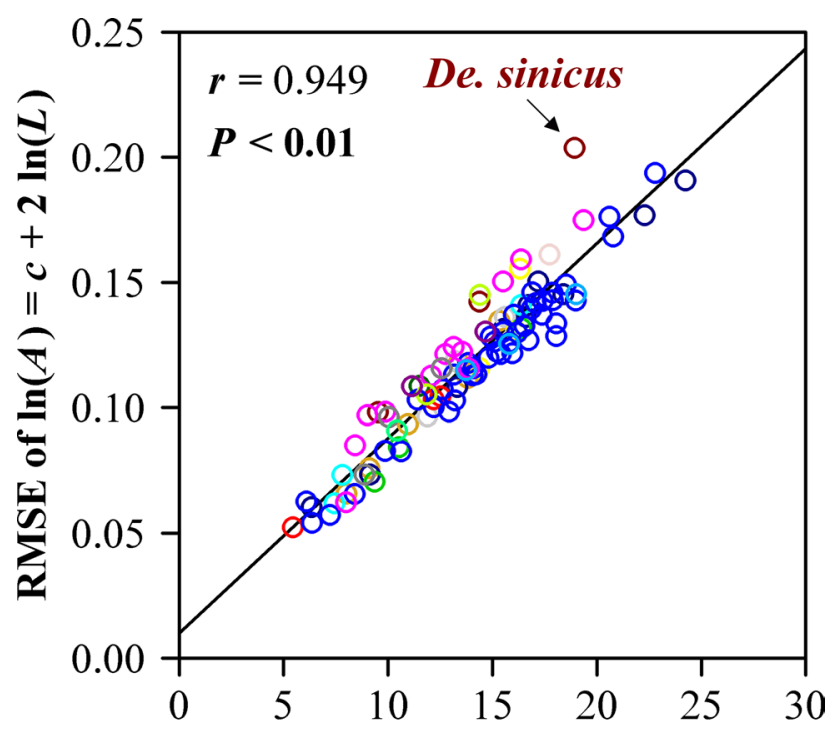

Coefficient of variation in $W / L(\%)$

Fig. 3 Correlation between the coefficient of variation of the ratio of leaf width to length and the root-mean-square error of a linear fit with a fixed slope 2 to the data of leaf area vs. length. Different colors for the open circles represent different genera of Bambusoideae. The data point that strongly deviated from the regression line corresponds to Dendrocalamus sinicus L.C. Chia et J. L. Sun

deviated from the regression line of RMSE for the power law function with a scaling exponent of 2 for leaf area and length vs. the coefficient of variation in $W / L$ ratio. This bamboo species has a narrow geographical distribution limited to three counties in southwestern Yunnan Province (China) adjacent to Myanmar, where there is a higher daily mean temperature and annual precipitation, compared to Nanjing of Jiangsu Province, where most bamboo species (89 bamboo taxa) were collected. Thus, further research is needed to explore the effects of climatic factors on the scaling exponent of leaf area vs. length. Nevertheless, the proportional relationship between leaf area and the product of leaf length and width appears to be more stable regardless of environmental factors.

Why bamboo leaves follow this consistent scaling relationship can only be speculated. One reason might be that bamboo leaves have parallel venation pattern typical for monocots. The proportional relationship between leaf $A$ and $L W$ might be related to the hydraulic performance of leaves (Brodribb et al. 2010). It is likely that the scaling relationship between $A$ and $L W$ in bamboo leaves reflects an evolutionary adaptation to achieve an optimal hydraulic performance and photosynthetic capacity. Relatively longer blades require more support investments; however, too broad blades render the parallel vein system useless (Brodribb et al. 2010). Thus, there should be a trade-off between the change in relative leaf width and concomitant modification in the hydraulic resistance such that leaf photosynthetic potential of parallel-veined leaves is maximized for a limited range of leaf width to length ratios.

We demonstrated that whether the leaf length could be used as a single predictor to calculate leaf area mainly depended on the extent of the variation in $W / L$ ratio. Although our leaves were sampled from three different provinces of China, the pooled data of $A$ vs. $L W$ on the $\log -\log$ plot exhibited a very strong linear relationship (Fig. 1b). This implies that the proportional relationship between leaf area and the product of leaf length and width 
is evolutionarily stable within Bambusoideae. The leaf area is approximately equal to $70 \%$ of the product of leaf length and width. However, this proportionality coefficient (i.e., the Montgomery parameter) does not apply to other species; it has been found to vary from $1 / 2$ to $\pi / 4$ for different leaf shapes (Shi et al. 2019a; Yu et al. 2020). Regarding the principle of similarity proposed by Thompson (1917) that states that there is a proportionality between the area of an object and its length squared, our results indicated that this postulate lacks generality. Although the Montgomery equation accurately described the relationship between $A$ and $L W$, Thompson (1917) principle of similarity held true only when there was a small intraspecific variation in the $W / L$ ratio. It is worth further investigating whether the intraspecific variation in leaf shape can be significantly affected by biotic and abiotic factors (e.g., climatic variables and interspecific competition for light, soil nutrients, and water). In that case, the estimated proportionality coefficients observed across different gradients or treatments can be used to quantify the responses of plants to given environmental stresses. In addition, if there is a small variation in $W / L$ ratio, we can calculate leaf area only using leaf length as a single predictor.

Author contribution statement JS and PS conceived this study; the manuscript was written by PS, YL, ÜN, EO and JS; PS and ÜN analyzed the data and contributed equally to this work. All authors read and commented on this manuscript.

Acknowledgements We thank the staff and students of Bamboo Research Institute of Nanjing Forestry University for publishing the raw data that we could use here. PS was supported by the Jiangsu Government Scholarship for Overseas (No: JS-2018-038), and JS was supported by the Deutsche Forschungsgemeinschaft (DFG) with a research scholarship (No: SCHR1672/1-1).

Funding Open Access funding enabled and organized by Projekt DEAL.

\section{Compliance with ethical standards}

Conflict of interest The authors declare that they have no conflict of interest.

Open Access This article is licensed under a Creative Commons Attribution 4.0 International License, which permits use, sharing, adaptation, distribution and reproduction in any medium or format, as long as you give appropriate credit to the original author(s) and the source, provide a link to the Creative Commons licence, and indicate if changes were made. The images or other third party material in this article are included in the article's Creative Commons licence, unless indicated otherwise in a credit line to the material. If material is not included in the article's Creative Commons licence and your intended use is not permitted by statutory regulation or exceeds the permitted use, you will need to obtain permission directly from the copyright holder. To view a copy of this licence, visit http://creativecommons.org/licenses/by/4.0/.

\section{References}

Brodribb TJ, Feild TS, Sack L (2010) Viewing leaf structure and evolution from a hydraulic perspective. Funct Plant Biol 37:488-498. https://doi.org/10.1071/FP10010

Gielis J (2003) A generic geometric transformation that unifies a wide range of natural and abstract shapes. Am J Bot 90:333-338. https:// doi.org/10.3732/ajb.90.3.333

Lin SY, Zhang L, Reddy GVP, Hui C, Gielis J, Ding YL, Shi PJ (2016) A geometrical model for testing bilateral symmetry of bamboo leaf with a simplified Gielis equation. Ecol Evol 6:6798-6806. https:// doi.org/10.1002/ece3.2407

Lin SY, Niklas KJ, Wan YW, Hölscher D, Hui C, Ding YL, Shi PJ (2019) Leaf data of 101 species, varieties, forms, and cultivars of bamboo. Dryad Dataset. https://doi.org/10.5061/dryad.905qfttgb

Lin SY, Niklas KJ, Wan YW, Hölscher D, Hui C, Ding YL, Shi PJ (2020) Leaf shape influences the scaling of leaf dry mass vs. area: a test case using bamboos. Ann Forest Sci 77:11. https://doi.org/10.1007/ s13595-019-0911-2

Milla R, Reich PB (2007) The scaling of leaf area and mass: the cost of light interception increases with leaf size. Proc R Soc Biol Sci 274:2109-2114

Montgomery EG (1911) Correlation studies in corn. In: Annual Report no. 24. Agricultural Experimental Station, Lincoln, NB, pp 108-159

Niinemets Ü, Portsmuth A, Tobias M (2007) Leaf shape and venation pattern alter the support investments within leaf lamina in temperate species: a neglected source of leaf physiological differentiation? Funct Ecol 21:28-40. https://doi.org/10.111 $1 /$ j.1365-2435.2006.01221.x

Niklas KJ, Cobb ED, Niinemets Ü, Reich PB, Sellin A, Shipley B, Wright IJ (2007) 'Diminishing returns' in the scaling of functional leaf traits across and within species groups. Proc Natl Acad Sci USA 104:8891-8896. https://doi.org/10.1073/pnas.0701135104

Peppe DJ, Royer DL, Gariglino B, Oliver SY, Newman S, Leight E, Enikolopov G, Fernandez-Burgos M, Herrera F, Adams JM et al (2011) Sensitivity of leaf size and shape to climate: Global patterns and paleoclimatic applications. New Phytol 190:724-739. https:// doi.org/10.1111/j.1469-8137.2010.03615.x

R Core Team (2015) R: a language and environment for statistical computing. R Foundation for Statistical Computing, Vienna, Austria. http://www.r-project.org/

Shi PJ, Xu Q, Sandhu HS, Gielis J, Ding YL, Li HR, Dong XB (2015) Comparison of dwarf bamboos (Indocalamus sp.) leaf parameters to determine relationship between spatial density of plants and total leaf area per plant. Ecol Evol 5:4578-4589. https://doi.org/10.1002/ ece3.1728

Shi PJ, Ratkowsky DA, Li Y, Zhang LF, Lin SY, Gielis J (2018) General leaf-area geometric formula exists for plants. Evidence from the simplified Gielis equation. Forests 9:714. https://doi.org/10.3390/ f9110714

Shi PJ, Liu MD, Ratkowsky DA, Gielis J, Su JL, Yu XJ, Wang P, Zhang LF, Lin ZY, Schrader J (2019a) Leaf area-length allometry and its implications in leaf-shape evolution. Trees Struct Funct 33:10731085. https://doi.org/10.1007/s00468-019-01843-4

Shi PJ, Liu MD, Yu XJ, Gielis J, Ratkowsky DA (2019b) Proportional relationship between leaf area and the product of leaf length width of four types of special leaf shapes. Forests 10:178. https://doi. org/10.3390/f10020178

Su JL, Niklas KJ, Huang WW, Yu XJ, Yang YY, Shi PJ (2019) Lamina shape does not correlate with lamina surface area: an analysis based on the simplified Gielis equation. Glob Ecol Conser 19:e00666. https://doi.org/10.1016/j.gecco.2019.e00666 
Thompson DW (1917) On growth and form. Cambridge University Press, London

Verwijst T, Wen DZ (1996) Leaf allometry of Salix viminalis during the first growing season. Tree Physiol 16:655-660

Wright IJ, Reich PB, Westoby M, Ackerly DD, Baruch Z, Bongers F, Villar R (2004) The worldwide leaf economics spectrum. Nature 428(6985):821-827. https://doi.org/10.1038/nature02403
Yu XJ, Shi PJ, Schrader J, Niklas KJ (2020) Nondestructive estimation of leaf area for 15 species of vines with different leaf shapes. Am J Bot. https://doi.org/10.1002/ajb2.1560

Publisher's Note Springer Nature remains neutral with regard to jurisdictional claims in published maps and institutional affiliations.

\section{Affiliations}

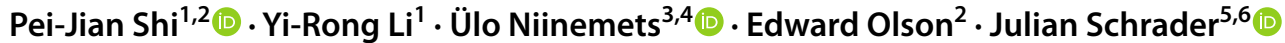

1 Bamboo Research Institute, College of Biology and the Environment, Nanjing Forestry University, Longpan Road 159, Nanjing 210037, China

2 Tropical Silviculture and Forest Ecology, University of Göttingen, Göttingen, Germany

3 Institute of Agricultural and Environmental Sciences, Estonian University of Life Sciences, Kreutzwaldi 1, 51006 Tartu, Estonia
4 Estonian Academy of Sciences, Kohtu 6, 10130 Tallinn, Estonia

5 Biodiversity, Macroecology and Biogeography, University of Göttingen, Göttingen, Germany

6 Department of Biological Sciences, Macquarie University, Sydney, NSW 2109, Australia 